\title{
Structural modulation of gut microbiota during alleviation of non-alcoholic fatty liver disease with Gynostemma pentaphyllum in rats
}

\author{
Shu-Hua Shen ${ }^{1}$, Ting-Yan Zhong ${ }^{2}$, Cui Peng ${ }^{2}$, Jie Fang ${ }^{3}$ and Bin Lv ${ }^{4^{*}}$
}

\begin{abstract}
Background: The current work aimed to assess whether Gynostemma pentaphyllum (GP), a Chinese herbal medicine, structurally modifies the gut microbiota in rats during non-alcoholic fatty liver disease (NAFLD) treatment.

Methods: High-fat diet (HFD)-induced NAFLD rats were orally administered water decoction of GP or equal amounts of distilled water per day for 4 weeks. Liver tissues were examined by histopathological observation, while intestinal tissues were examined by both histopathological and ultrastructural observations. The levels of fasting blood glucose (FBG), fasting serum insulin (FINS), total cholesterol (TC), triglycerides (TG), high-density lipoprotein cholesterol (HDL-C), low-density lipoprotein cholesterol (LDL-C), alanine transaminase (ALT) and aspartate transaminase (AST) were measured by enzymatic method. The levels of toll-like receptor 4 (TLR-4), tumor necrosis factor-alpha (TNF-a), interleukin-1-beta (IL-1 $\beta$ ) and interleukin-6 (IL-6) in both serum and hepatic tissues were measured by RT-qPCR. The protein expression level of TLR-4 in hepatic tissues was detected by western blot. The gut microbiota was assessed by 16S rRNA-based microbiota analysis.

Results: GP maintained intestinal integrity and reversed gut dysbiosis in high-fat diet (HFD)-induced NAFLD rats. This also reduced the ratio of Firmicutes to Bacteroidetes, enriching the abundance of beneficial bacteria (Lactococcus spp.) and inhibiting the abundance of pathogenic bacteria (Ruminococcus spp.) in the gut. The levels of pro-inflammatory cytokines (TNF- $a, I L-1 \beta$ and IL-6) and the expression of TLR4 were downregulated $(P<0.05)$, while the insulin resistance index, HOMA-IR showed improvement by GP treatment $(P<0.05)$. Liver function indicators (ALT and AST) were remarkably decreased $(P<0.01)$. Besides, GP treatment reduced TG and LDL-C levels $(P<0.05)$, and increased HDL-C level $(P<0.05)$ compared with NAFLD group.
\end{abstract}

Conclusion: The structural alterations of gut microbiota induced by GP are associated with NAFLD alleviation. Keywords: Non-alcoholic fatty liver disease, Gynostemma pentaphyllum, Gut microbiota, Endotoxemia, Insulin resistance

\footnotetext{
* Correspondence: ssh@zcmu.edu.cn

${ }^{4}$ Department of Gastroenterology, The First Affiliated Hospital of Zhejiang

Chinese Medical University, 54 Youdian Road, Zhejiang 310006, Hangzhou,

China

Full list of author information is available at the end of the article
}

(c) The Author(s). 2020 Open Access This article is distributed under the terms of the Creative Commons Attribution 4.0 International License (http://creativecommons.org/licenses/by/4.0/), which permits unrestricted use, distribution, and reproduction in any medium, provided you give appropriate credit to the original author(s) and the source, provide a link to the Creative Commons license, and indicate if changes were made. The Creative Commons Public Domain Dedication waiver (http://creativecommons.org/publicdomain/zero/1.0/) applies to the data made available in this article, unless otherwise stated. 


\section{Background}

Nonalcoholic fatty liver disease (NAFLD) is defined as the presence of significant hepatic lipid accumulation (at least in $5 \%$ of hepatocytes) in the absence of competing liver disease etiologies, including chronic viral or autoimmune hepatitis, Wilson's disease, and heavy alcohol consumption $[1,2]$. NAFLD represents a group of ailments, ranging between simple steatosis and non-alcoholic steatohepatitis (NASH), which at times lead to cirrhosis [3]. It is considered one of the main factors causing liver disease worldwide, with a prevalence of around 25-45\% [4]. In addition, NAFLD is increasingly recognized as the liver disease component of metabolic syndrome (MetS) [5], and is associated with a broad spectrum of diseases including obesity, type 2 diabetes (T2D), hyperlipidemia, hypertension, cardiovascular disease (CVD), and cancer $[6,7]$. As a result, NAFLD patients have an increased risk for both liver and MetS morbidity and mortality [8], causing substantial economic and clinical burden to society [9].

NAFLD pathogenesis remains incompletely understood. The "multiple hit" hypothesis provides a relatively accurate explanation, wherein multiple insults act jointly in individuals with genetic predisposition to trigger NAFLD as well as associated complications. Such hits include insulin resistance, nutrition, gut microbiome, hormones secreted by the fat tissues, and genetic and epigenetic factors [10]. Recently, considerable evidence suggests gut microbiome dysbiosis has a critical function in NAFLD progression [11]. Dysbiosis increases gut permeability to bacterial products, promotes energy absorption, aggravates insulin resistance (IR), facilitating systemic bacterial translocation and hepatic inflammation [12].

Traditional Chinese Medicine has been employed in Asia for many thousand years [13]. Much attention has been paid to its remarkable efficacy and low side effects in treating MetS, especially NAFLD [14, 15]. Gynostemma pentaphyllum $(G P)$, one of the popular herbs, has been used in traditional medicine since ancient times in treating various diseases, such as hyperlipemia, hyperglycemia, hepatitis, gastroenteritis [16]. We and others have demonstrated that GP has functions in protecting against NAFLD and other components of MetS $[17,18]$. Cell culture and animal models have indicated the important role of GP in lipid reduction, glucose regulation $[19,20]$, as well as liver protection [21, 22]. Our preliminary study in high-fat diet (HFD) rats demonstrated that GP can effectively reduce blood lipid levels and protect liver function, negatively correlating with the content of gypenoside (saponins isolated from $G P)$. However, it remains unclear regarding the mechanism of how GP alleviates NAFLD.

Hence, in this study, we explored the effect of GP on NAFLD (HFD induced) rats using dilinoleoyl phosphatidylcholine $(D L P C)$ as a contrast $[23,24]$.

\section{Methods}

\section{Drug preparation}

The GP material was obtained from the First Affiliated Hospital of Zhejiang Chinese Medical University (purchased from Huadong Medicine Co., Ltd., Date of Production: 20160706, Hangzhou, China) and identified by the Director of traditional Chinese pharmacy of the hospital, Mrs. Wen-Xia Zheng. GP was decocted twice with $4000 \mathrm{ml}$ deionized water, $1 \mathrm{~h}$ each time, followed by concentrating it to $2 \mathrm{~g}$ per milliliter $(2 \mathrm{~g} / \mathrm{ml})$ under normal pressure and making into a liquid extract. The quality of the GP was controlled by HPLC -MS analysis (Fig. 1). The collection, processing and usage of GP were performed according to the Guidelines for clinical use of the Pharmacopoeia of the People's Republic of China.

\section{Animal experiments}

\section{Experimental animals}

The rats were bought from Zhejiang Laboratory Animal Center (Production License Number: SCXK (Zhe)20140001, Use License Number: SYXK (Zhe)2014-0008). Animal experiments were approved and performed in accordance with the guidelines of the Ethics Committee of the first

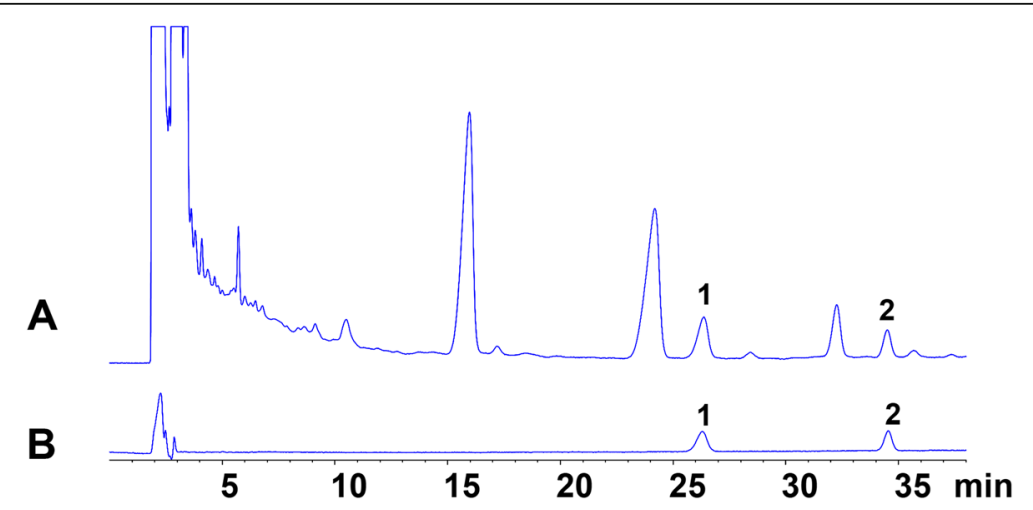

Fig. 1 High-performance liquid chromatography chromatogram of sample (a) and standard (b) solutions. 1: Ginsenoside Rb3, 2: Ginsenoside Rd. 
affiliated hospital of Zhejiang Chinese medical University, Hangzhou, China (Approval Number: 2017-k-098). Sixty male adult pathogen-free Sprague-Dawley (SD) rats, weighing 180-220 g were selected for this study. The rats were randomly divided into control group $(n=10$, Control), model group $(n=10, \mathrm{NAFLD})$, positive drug group $(n=10$, DLPC), high dosage group $(n=10, \mathrm{GPH})$, middle dosage group $(n=10, \mathrm{GPM})$ and low dose group $(n=10, \mathrm{GPL})$.

\section{Molding}

The rats were acclimatized to the housing conditions for 7 days, with free access to water and standard chow diet in laboratory conditions $\left(18-24{ }^{\circ} \mathrm{C}\right.$, day-night cycle of $12 \mathrm{~h}$ with light changes at 6:00 am and 6:00 pm). After that, the rats were fed with chow diet or high-fat diet for another 4 weeks before drug administration. Animals in the control group were administered with standard chow consisting of $67 \%$ carbohydrate, $10 \%$ fat, $23 \%$ protein, providing a total calorie of $3.6 \mathrm{kcal} / \mathrm{g}$. While the animals in the model group, the positive drug group and the high, middle and low dosage groups were continuously fed on HFD consisting of $52 \%$ carbohydrate, $30 \%$ fat, $18 \%$ protein, which provided a total calorie of $4.8 \mathrm{kcal} / \mathrm{g}$ [25]. According to our previous studies and preliminary experiments, indices such as body weight, intake and activities, fasting blood glucose (FBG), total cholesterol (TC), triglycerides (TG), high-density lipoprotein cholesterol (HDL-C), low-density lipoprotein cholesterol (LDL-C), alanine transaminase (ALT) and aspartate transaminase (AST) to confirm the success of model establishment.

\section{Drug administration}

From week 5, the GP treatment groups were orally administered water decoction of GP at concentrations of 6 $\mathrm{g} \cdot \mathrm{kg}^{-1} \cdot \mathrm{d}^{-1}, 3 \mathrm{~g} \cdot \mathrm{kg}^{-1} \cdot \mathrm{d}^{-1}$ and $1.5 \mathrm{~g} \cdot \mathrm{kg}^{-1} \cdot \mathrm{d}^{-1}$ once a day for another 4 weeks based on the conversion formula between human per $70 \mathrm{~kg}$ and rats per $200 \mathrm{~g}$ (1: 0.018) and our preliminary experiments. The rats in the positive drug group were orally administered $D L P C$ at a concentration of $22.8 \mathrm{mg} \cdot \mathrm{kg}^{-1} \cdot \mathrm{d}^{-1}$, while the control group and the model group were administered equal amounts of distilled water per day for 4 weeks.

\section{Specimen collection}

After 8 weeks of HFD, the animals were fasted for $8 \mathrm{~h}$. Next day, blood was drawn from the caudal vein, and then the rats were euthanized by cervical dislocation. The blood was centrifuged at $3000 \mathrm{rpm}$ for $15 \mathrm{~min}$, and the serum was stored at $-80^{\circ} \mathrm{C}$. After laparotomy, the liver, the contents from the cecum and the ileum and the colon besides the cecum were removed. The contents (about $1.0 \mathrm{~g}$ ) of the cecum from each rat were taken, maintained in tubes with glass beads, and stored at $-80^{\circ} \mathrm{C}$. Part of the liver and the colon and terminal ileal tissues were fixed with neutral formaldehyde, and then the paraffin slices were prepared. Others were stored at $-80^{\circ} \mathrm{C}$.

\section{Biochemical analyses}

Biochemical assessments of serum lipids, glucose, enzymes, insulin and cytokines were performed in the central laboratory (The first affiliated hospital of Zhejiang Chinese medical University, Hangzhou, China).

The index of lipids in the serum, including TC, TG, HDL-C and LDL-C and the marker enzymes for liver damage and disease, including ALT and AST were measured by commercial enzymatic kits (Jiancheng Bioengineering Inst., Nanjing, China).

The index of fasting serum insulin (FINS), tumor necrosis factor-alpha (TNF- $\alpha$ ), interleukin-1-beta (IL-1 $\beta$ ) and interleukin-6 (IL-6) levels were measured by commercial ELISA kits (MultiSciences, Shanghai, China).

FBG was determined with a glucose meter (Sinocare, Changsha, China) by collecting the blood from the tip of the tail vein. Homeostasis model assessment of insulin resistance (HOMA-IR) was calculated using the formula: fasting glucosex fasting insulin/22.5 [26].

\section{Histopathology observation \\ HE staining}

Liver or intestinal mucosal specimens were formalinfixed, paraffin-embedded, and cut into sections (4- $\mu \mathrm{m}$ thickness), which underwent staining with hematoxylin (Servicebio, Wuhan, China; $5 \mathrm{~min}$ ) and eosin (Servicebio; $2 \mathrm{~min}$ ). A light microscope (Nikon, Tokyo, Japan) was employed for observations, with 8 sections assessed per rat.

\section{ORO staining}

Liver cryosections (thickness, $6 \mu \mathrm{m}$ ) underwent staining with ORO (Servicebio) for $20 \mathrm{~min}$, and then counterstained with hematoxylin (Servicebio) for $1 \mathrm{~min}$. A light microscope (Nikon) was employed for observations, with 8 sections assessed per rat.

\section{Ultrastructural observation}

Intestinal mucosal sections were fixed in $2.5 \%$ glutaraldehyde (Sinopharm Chemical Reagent Co., Ltd., Shanghai, China) for $24 \mathrm{~h}$ and $1 \%$ osmic acid (Sinopharm Chemical Reagent Co., Ltd) for $2 \mathrm{~h}$, dehydrated through a graded series of ethanol (Sinopharm Chemical Reagent Co., Ltd) and acetone solution (Sinopharm Chemical Reagent Co., Ltd), embedded in Epon812 (Servicebio), sectioned with ultramicrotome (Leica, Weztlar, Germany), and stained with uranyl acetate (Sinopharm Chemical Reagent Co., Ltd) followed by lead citrate (Sinopharm Chemical Reagent Co., Ltd), then observed with transmission electron 
microscopy (Hitachi, Tokyo, Japan). A total of 20 tissue sections were analyzed for each animal.

\section{Real-time quantitative reverse-transcription $P C R$}

Fifty mg liver tissue was weighed, and then the mRNA expression levels of toll-like receptor 4 (TLR-4), TNF- $\alpha$, IL- $1 \beta$ and IL- 6 were detected by quantitative real-time reverse-transcription PCR (Additional file 1: Table S5) according to the previously published RT-qPCR methods [27].

\section{Western blotting (WB)}

Twenty mg liver tissue was weighed, and the protein expression level of TLR- 4 was detected by WB as described previously [28].

\section{Gut microbiota analysis}

Fecal specimens upon snap-freezing in liquid nitrogen were stored at $-80^{\circ} \mathrm{C}$. DNA extraction was carried out by the CTAB protocol. For cecal fecal specimens, the 16S rRNA gene encompassing V3-V5 regions, 18S rRNA V4 and V9 regions, ITS1 and ITS2 regions underwent amplification with Phusion ${ }^{\circ}$ High-Fidelity PCR Master Mix in GC Buffer (New England Biolabs). TruSeq ${ }^{\circ}$ DNA PCR-free sample preparation kit was used to prepare a library. Microbial sequencing was performed on the HiSeq2500 Illumina platform (PE250). High quality reads were obtained (QIIME V1.9.1) and underwent clustering into OTUs on the basis of 97\% sequence similarity (Uparse v7.0.1001). The nearest alignment space termination multi-aligner was employed for aligning high-quality sequences based on SILVA compatible database alignment, removing non-aligned reads. Chimeric sequences detected by the UCHIME algorithm were also excluded. Read classification used a Bayesian classifier based on the RDP database generated by our team. We removed all reads not classifiable at the kingdom level. For alpha diversity assessment, rarefaction analysis and Shannon index calculation were carried out with QIIME. Fast UniFracPCoA was carried out via phylogenetic tree construction and by inserting the representatives of various OTUs, also with QIIME [29]. As proposed previously [30], whether separation among animal groups in the principal coordinate analysis (PCoA) score plot is statistically significant was evaluated by multivariable analysis of variance test with statistically significant differences in physiological/biochemical parameters.

\section{Statistical analysis}

Ten-replicate assays were presented as mean \pm standard deviation (SD). Differences in biochemical indicators were examined by unpaired two-tailed Student's t-test. Multiple groups were compared by one-way ANOVA with Newman-Keuls post hoc test. Nextgeneration sequencing data were examined by the Tukey's test. $P<0.05$ indicated statistical significance. In figures, different superscript letters indicate significant differences (post hoc ANOVA). R 3.4.3 was used for statistical analysis.

\section{Results \\ GP prevents HFD-induced hyperlipidemia of NAFLD}

Previous studies showed that the rats fed a HFD produced high levels of TC, TG and LDL-C, while the production of HDL-C was reduced [31, 32]. Using a rat NAFLD model, we observed that the rats fed on HFD for 8 weeks led to significant differences in the lipids levels when compared with control group (Table 1). TC, TG and LDL-C levels were significantly increased in the serum of NAFLD rats when compared with control rats, whereas the HDL-C level was significantly reduced. Meanwhile, for the middle dose and high dose GP treated rats, the serum levels of TC, TG and LDL-C were also reduced significantly when compared with NAFLD rats. Significant increase in HDL levels was observed in the medium dose of GP (GPM) and high dose of $G P(\mathrm{GPH})$ rats. GP treatment demonstrated a dose-dependent effect, alleviating hyperlipidemia. DLPC treatment reduced the serum TG and LDL-C levels, and increased HDL-C level as expected. These findings suggested that GP prevents HFD-induced hyperlipidemia in rats (Table 1).

\section{GP prevents HFD-induced non-alcoholic fatty liver (NAFLD)}

Previous studies have shown that NAFLD rats produced high levels of marker enzymes for liver damage and disease, which included ALT and AST [31, 32]. The serum levels of the marker enzymes were measured. The results showed that ALT and AST in NAFLD rats were higher when compared with control

Table 1 GP prevents HFD-induced hyperlipidemia

\begin{tabular}{lllll}
\hline & $\mathrm{TC}(\mathrm{mmol} / \mathrm{L})$ & $\mathrm{TG}(\mathrm{mmol} / \mathrm{L})$ & $\mathrm{LDL}-\mathrm{C}(\mathrm{mmol} / \mathrm{L})$ & $\mathrm{HDL}-\mathrm{C}(\mathrm{mmol} / \mathrm{L})$ \\
\hline NAFLD & $2.14 \pm 0.26$ & $0.63 \pm 0.04$ & $1.61 \pm 0.10$ & $0.32 \pm 0.17$ \\
Control & $1.26 \pm 0.24^{* *}$ & $0.50 \pm 0.07^{* *}$ & $1.26 \pm 0.34^{*}$ & $0.47 \pm 0.15^{* *}$ \\
DLPC & $1.98 \pm 0.62$ & $0.51 \pm 0.08^{*}$ & $1.15 \pm 0.25^{* *}$ & $0.47 \pm 0.21^{*}$ \\
GPL & $1.87 \pm 0.33$ & $0.48 \pm 0.07^{* *}$ & $1.14 \pm 0.21^{* *}$ & $0.39 \pm 0.12$ \\
GPM & $1.69 \pm 0.28^{*}$ & $0.48 \pm 0.07^{* *}$ & $1.16 \pm 0.36^{* *}$ & $0.51 \pm 0.08^{* *}$ \\
GPH & $1.29 \pm 0.27^{* *}$ & $0.49 \pm 0.16^{* *}$ & $1.14 \pm 0.24^{* *}$ & $0.54 \pm 0.09^{* *}$ \\
\hline
\end{tabular}

Serum concentrations of TC, TG, LDL-C and HDL-C in each group compared with NAFLD group ( $n=10$ for each group). Data are shown as mean \pm standard deviation. Whereas * and ${ }^{* *}$ represent statistically significant results $(P<0.05, P<0.01$, respectively) based on Newman-Keuls post hoc one-way ANOVA analysis. GPL: low dose of GP treatment; GPM: middle dose of GP treatment; GPH: high dose of GP treatment 

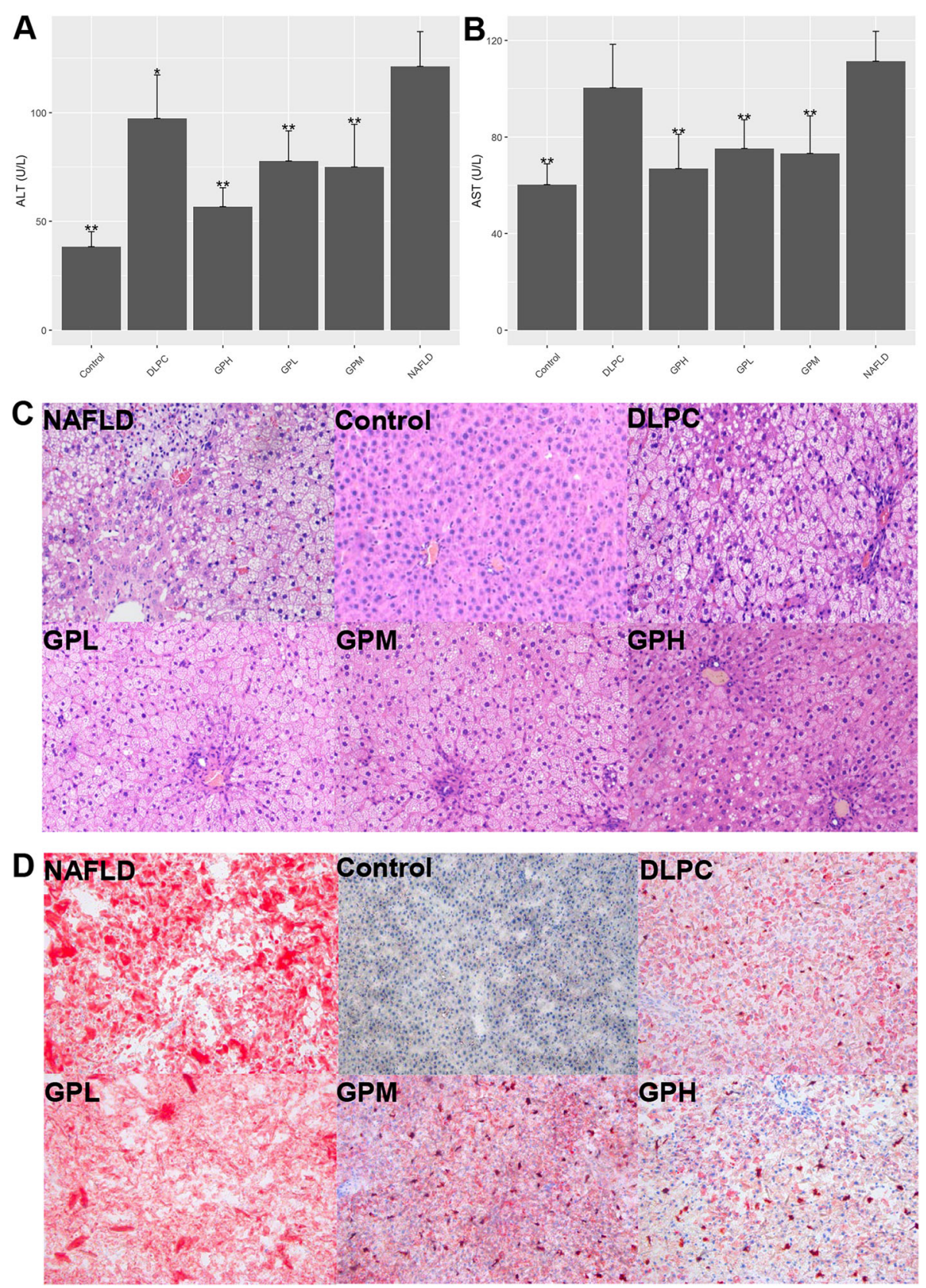

Fig. 2 GP prevents HFD-associated Non-alcoholic fatty liver disease (NAFLD) in the rat model. a, (b) Serum concentrations of ALT and AST in various groups compared with the NAFLD group ( $n=10$ for each group). Data are mean \pm standard deviation. ${ }^{*} P<0.05$; ${ }^{* *} P<0.01$ (one-way ANOVA with Newman-Keuls post hoc analysis). c H\&E staining of the liver tissue in the NAFLD, Control, DLPC, GPL, GPM and GPH groups (magnification, 200X) ( $n=10$ for each group). Major histopathological changes induced by HFD in rat liver included hepatosteatosis, ballooning and hiver inflammation. $\mathbf{d}$ Liver lipid content was determined by Oil-red O staining in the NAFLD, Control, DLPC, GPL, GPM and GPH groups (magnification, 200 $\times)$ ( $n=10$ for each group)

rats. While GP treatment reduced both ALT and AST levels significantly. DLPC improved ALT to some extent, but showed no remarkable effect on AST (Fig. 2a, b, Additional file 1: Table S1).

As expected, both H\&E staining and ORO staining revealed the normal structure of liver in control rats, while hepatic steatosis in NAFLD rats [31, 32]. However, the extent of steatosis in the liver of GP treated rats was remarkably reduced in a dose-dependent manner. $D L P C$ also alleviated hepatic steatosis to a certain extent (Fig. 2c, d). These findings suggested that GP prevents HFD-induced NAFLD in rats. 

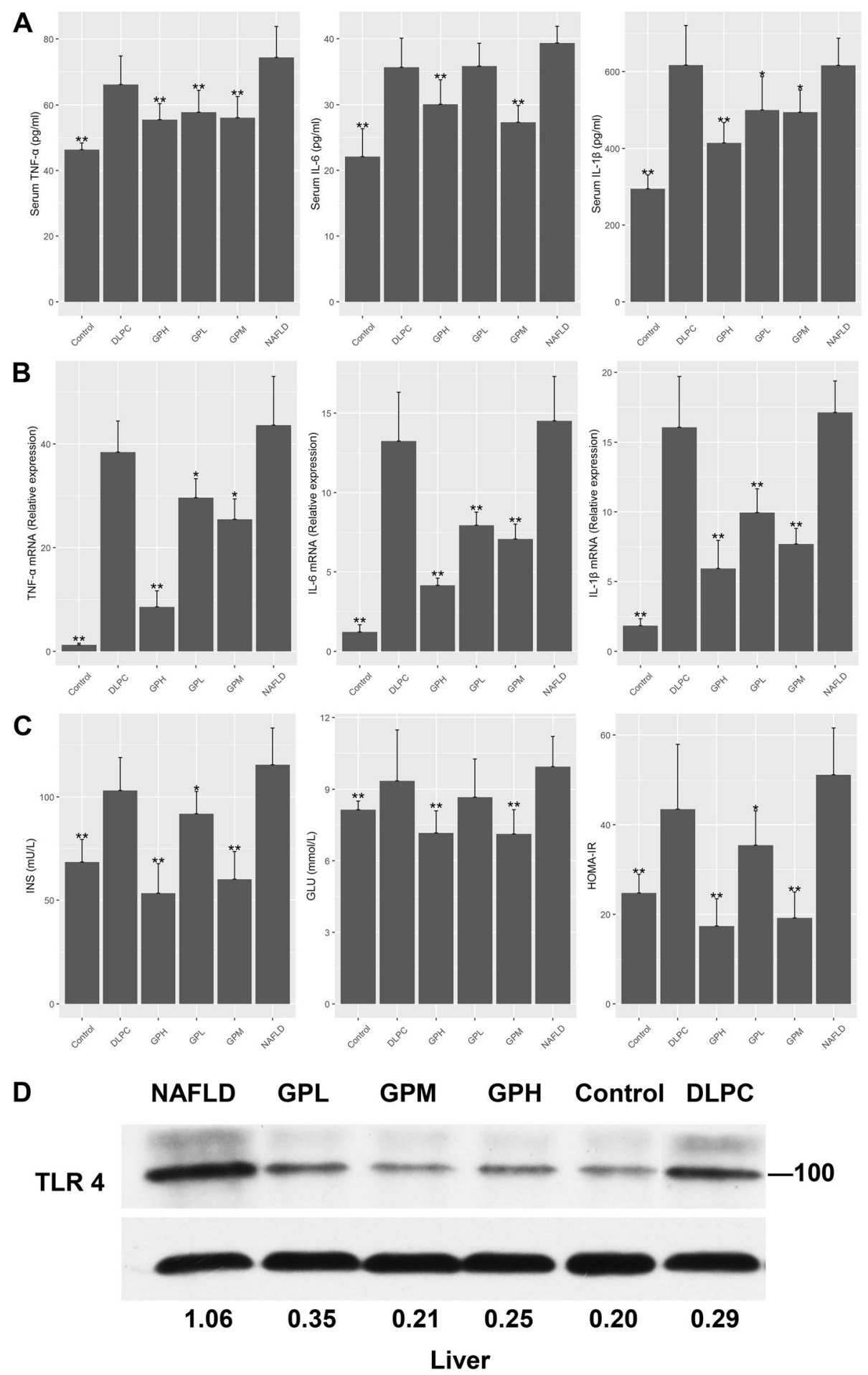

Fig. 3 GP prevents HFD-induced inflammation, endotoxemia and insulin resistance in rats. a Serum TNF- $\alpha$, IL-1 $\beta$ and IL-6 amounts in each group were compared with those of the NAFLD group ( $n=10$ for each group). b TNF- $a$, IL-1 $\beta$ and IL-6 mRNA levels in hepatic tissues as assessed by RTqPCR, compared with the NAFLD group ( $n=10$ for each group). c Serum concentrations of INS, GLU and HOMA-IR in each group were

compared with those of the NAFLD group ( $n=10$ for each group). Data are mean \pm standard deviation. ${ }^{*} P<0.05$; ${ }^{* *} P<0.01$ (one-way ANOVA with Newman-Keuls post hoc analysis). d Protein amounts were normalized to Actin expression, and the relative ratios based on the Control group are shown below the immunoblots 


\section{GP reduces inflammation, endotoxemia and insulin resistance}

Previous studies have shown that NALFD rats produce high amounts of pro-inflammatory cytokines in the liver and serum, e.g., TNF- $\alpha$, IL-1 $\beta$ and IL-6 [33]. We measured the above cytokines in rats following 8 weeks of HFD with or without GP or DLPC treatment. The results showed that TNF- $\alpha$, IL-1 $\beta$ and IL- 6 amounts were higher in serum and hepatic tissues of NAFLD rats compared with control animals. Moreover, GP treatment resulted in decreased TNF- $\alpha$, IL- $1 \beta$ and IL- 6 amounts, while $D L P C$ administration did not (Fig. 3a, b, Additional file 1: Table S2,S3).

"Metabolic endotoxemia" may promote inflammatory reactions and insulin resistance in HFD-fed rats through
TLR4 signaling [34, 35]. We examined the effects of GP and $D L P C$ on TLR4 protein amounts in the liver. Insulin resistance was estimated using HOMA-IR. The results showed that GP reduced endotoxemia and insulin resistance in HFD-fed rats, while DLPC did not (Fig. 3c, d, Additional file 1: Table S4). These findings suggested that GP reduced inflammation, endotoxemia and insulin resistance in NAFLD rats.

\section{GP maintains intestinal integrity}

Given that gut microbiota dysbiosis in HFD-fed rats might alter intestinal permeability and promote lipopolysaccharide (LPS) release into the blood stream [36], whether GP and $D L P C$ modulate intestinal barrier integrity was investigated.

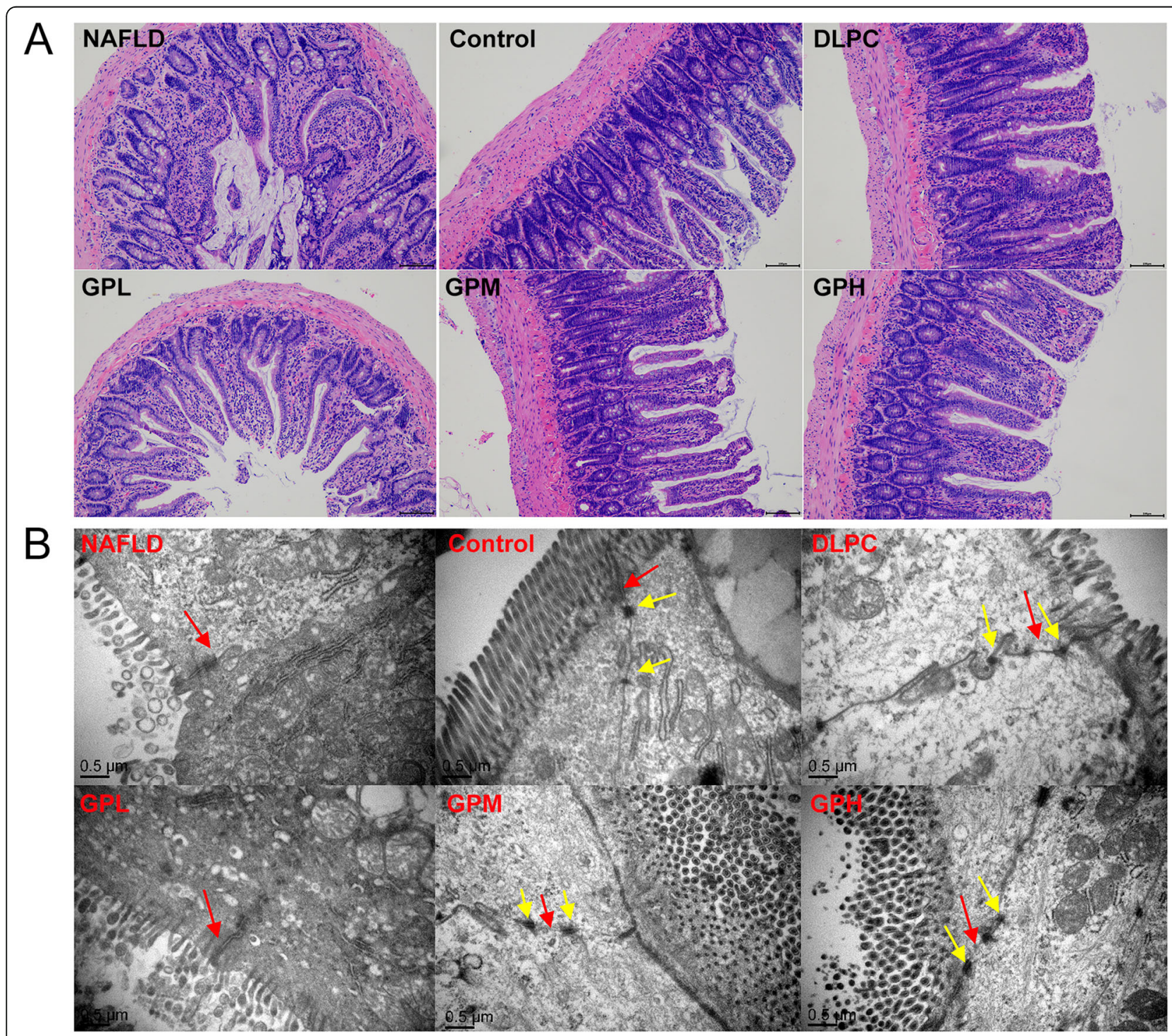

Fig. 4 GP maintains intestinal integrity in rats. a HE staining of ileal tissue in NAFLD, Control, DLPC, GPL, GPM and GPH groups (magnification, 100x) ( $n=10$ for each group). Major histopathological changes induced by HFD in rat ileum were sparse and irregular microvilli of epithelial cells with inflammatory cell infiltration, damaged tight junctions and desmosomes. b Transmission electron microscopy of ileum in NAFLD, Control, DLPC, GPL, GPM and GPH groups (magnification, 30,000x) ( $n=10$ for each group). Red arrow: tight junctions; Yellow arrow: desmosomes 

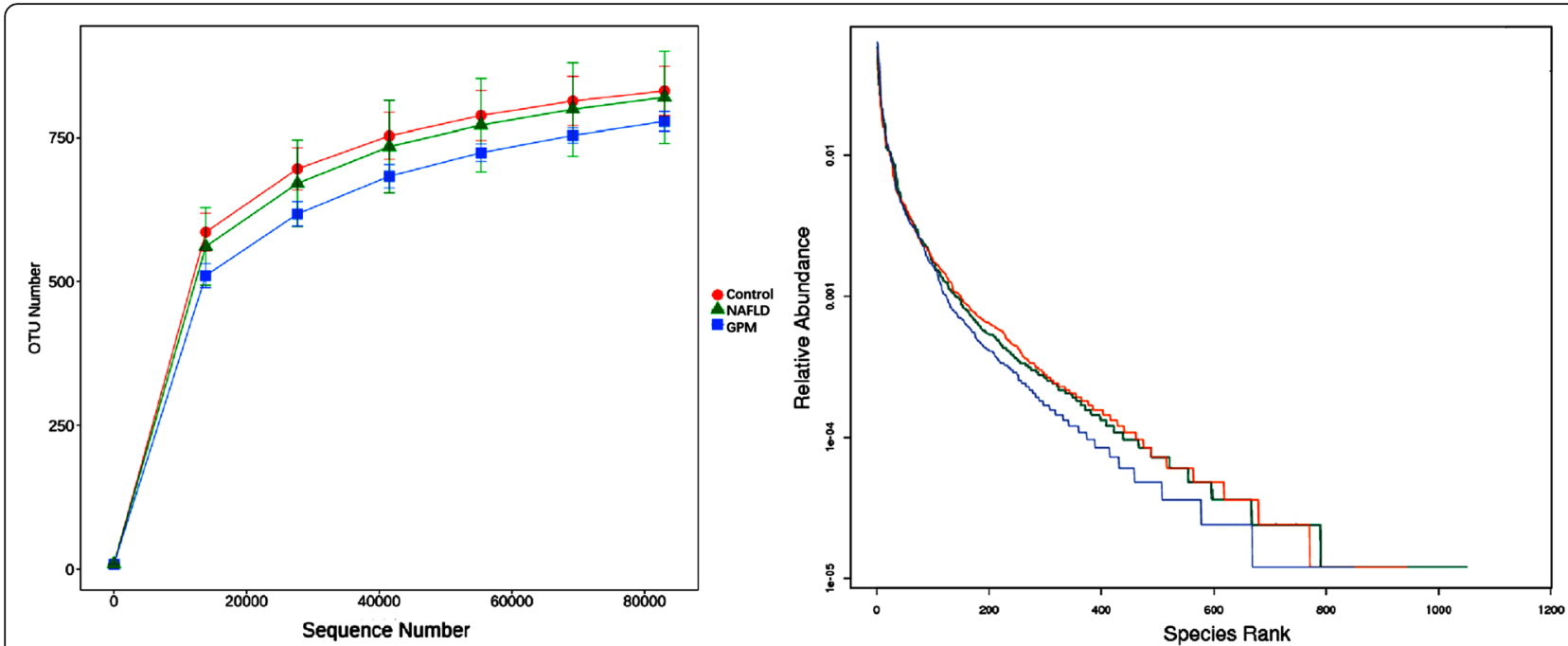

Fig. 5 Rarefaction diversity and Rank abundance curves. We examined the structural alterations of the gut microbiome in the NAFLD, Healthy control and GP middle dose groups, respectively, before and after GP treatment. Rarefaction diversity and Rank abundance curves showed that although rarefaction diversity curves showed no plateau, the diversity was mostly captured, indicating that the sequencing results could represent the bacterial diversity of the gut

In the control group, the microvilli of epithelial cells were rich and regular, and the tight junctions were clear and complete, with small gaps and abundant desmosomes. In the NAFLD group, the microvilli of epithelial cells were sparse and irregular, and the tight junctions were damaged, with large gaps and loose or few desmosomes. Compared with NAFLD group, the rats in GP and DLPC groups showed more regular microvilli, improved tight junctions and desmosomes, and smaller gaps. The former ones were dose-dependent, showing a better result than the latter (Fig. 4a, b). These findings suggested that GP might improve intestinal barrier integrity in NAFLD rats.

\section{The gut microbiome is structurally modulated by GP administration}

We next examined gut microbiome compositions in three animal groups, including the NAFLD, healthy control and GP middle dose groups, pre- and post-GP administration, respectively. A total of 750,000 acceptable raw sequences (34,753 unique sequences) and 3222 OTUs were detected in 15 samples, with averagely $874 \pm 101 /$ sample. Although rarefaction diversity curves showed no plateau, the diversity was mostly captured (Fig. 5). PCA analysis revealed that compared with the HFD-induced NAFLD model group, the GP treatment group showed closer deviation towards the healthy animals, suggesting that gut microbiota structure in rats showed divergence from baseline after a 4-week GP treatment (Fig. 7a). The Venn diagram indicated that GP treated and control rats shared more OTUs than NAFLD rats (Fig. 6). Unique OTUs in NALFD rats were triple the amounts in GP treated rats (Fig. 6). Shannon index analysis showed that intestinal flora density in the NAFLD group was significantly reduced, and GP treatment resulted in recovered intestinal flora diversity compared with control rats (Fig. 7b). The gut microbiota of rats consisted of Bacteroidetes, Firmicutes, Proteobacteria, Elusimicrobia, Cyanobacteria, Fusobacteria, Actinobacteria, Spirochaetes and Verrucomicrobia at the phylum level. Of these, Bacteroidetes were the most abundant organisms, followed by Firmicutes and Proteobacteria (Fig. 7c). Several studies have indicated that

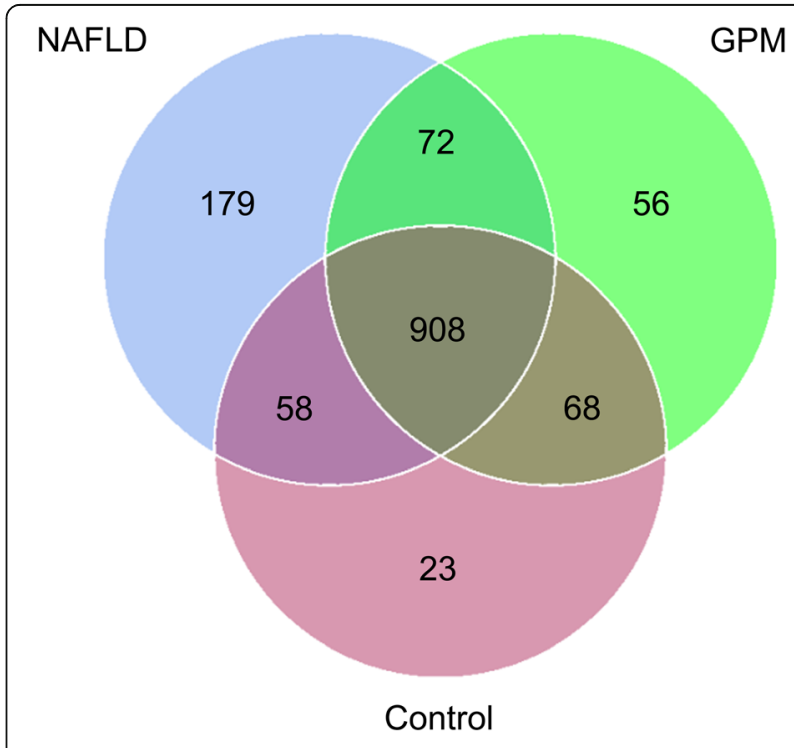

Fig. 6 Venn plot of the number of OTUs in each group 


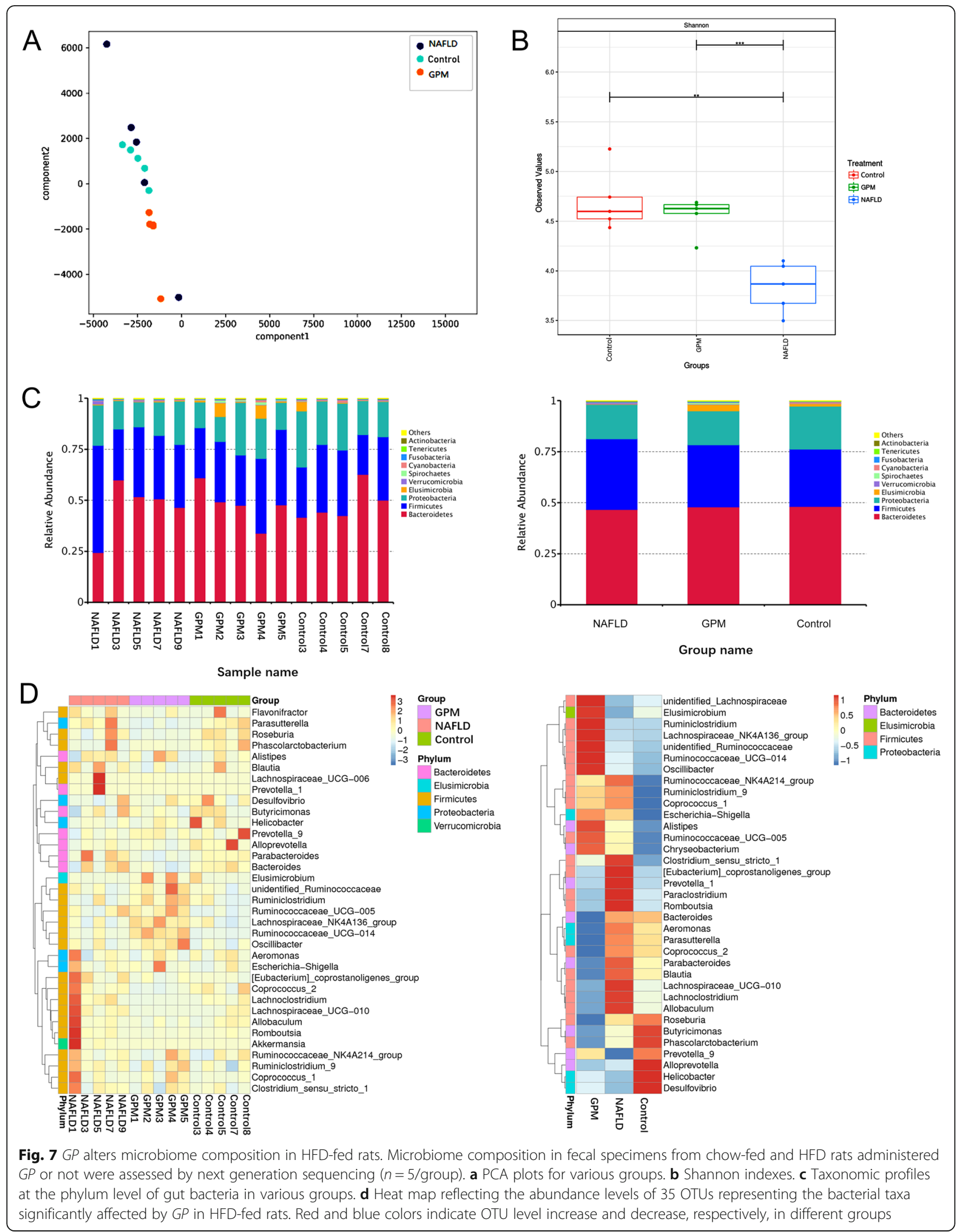


elevated Firmicutes to Bacteroidetes (two main phyla) ratio is associated with lipid metabolism disorder [37-39]. Lipid metabolism has an important function in NAFLD [40]. Namely, GP treatment (GPM) decreased the Firmicutes-to-Bacteroidetes ratio in HFD-fed (NAFLD) rats to a value comparable to that of control rats (Fig. $7 d$ ). The abundance levels of Elusimicrobia and Cyanobacteria were both higher in GP and control rats in comparison with HFD-fed rats (Fig. 7d). Besides, many bacterial species showed increased amounts after GP administration in comparison with the model group, indicating that $G P$ may enrich specific bacterial species (Fig. 7d). Moreover, GP treatment increased the abundance levels of beneficial bacteria such as Lactococcus spp. and decreased those of the pathogenic bacteria, including Ruminococcus spp. (Fig. 7d). Collectively, these results showed that GP modulated the gut microbiome in HFD-fed rats, yielding a microbiome composition comparable to that of control rats.

\section{Discussion}

Traditional Chinese Medicine has been used for treating several diseases for thousands of years in China. Nevertheless, Chinese herbs are highly complex with undefined mechanisms, which prevents the identification of active chemical components. Many studies have suggested that Chinese herbs prevent or alleviate diseases by controlling gut microbiome structure [41-44]. This research may provide a potential mechanism as to how GP alleviates NAFLD.

As shown above, GP administration exerted concentration-dependent effects in HFD-induced NAFLD rats. GP treated rats showed significant improvement in liver health by lowering ALT and AST levels, and decreasing hepatic steatosis. Besides, we observed an improvement in hyperlipidemia and hyperglycemia in GP treated animals, corroborating a previous Australian study [45]. These data showed that GP can alleviate NAFLD and other components of MetS, making it a promising candidate medicine for NAFLD and the associated complications.

The current model of HFD-induced NAFLD is largely explained by gut microbiota dysbiosis [46]. Pathogenic bacteria are excessively grown, producing too much LPS that exceeds hepatic clearance capacity, and therefore, "metabolic endotoxemia" [47]. After activation by LPS, TLR4 stimulates the production of inflammatory kinases (such as JNK, IKK and p38), inhibits the phosphorylation of insulin receptor substrates, and damages the insulin signal transduction pathway, resulting in the development of IR and inflammation $[48,49]$. In other words, dysbiosis increases gut permeability to bacterial products and aggravates IR, facilitating systemic bacterial translocation and hepatic inflammation. As shown above, GP treatment improved gut barrier integrity, reduced inflammation, and alleviated endotoxemia and IR in NALFD rats. These beneficial effects might result from specific gut microbiome changes.

High-quality experimental and some human studies have confirmed the therapeutic effects of prebiotics and probiotics on NAFLD, via modulation of the gut microbiota [50]. However, no gut microbiota modulation by GP has been reported to date. Previous studies have found that HFD increases the ratio of Firmicutes to Bacteroidetes in NALFD rats [51]. The present study confirmed these findings and demonstrated that GP might exert anti-NAFLD effects by lowering the Firmicutes-to-Bacteroidetes ratio. Besides, the abundance levels of several beneficial bacteria such as $\mathrm{Lac}$ tococcus spp. were increased by GP; in contrast, the abundance levels of pathogenic bacteria, such as Ruminococcus spp., were decreased. Lactococcus was found to be reduced by HFD, and is negatively correlated with NAFLD $[52,53]$. Ruminococcus ferments complex carbohydrates such as cellulose, pectin, resistant starch etc. in some cases could be pro-inflammatory [54]. Meanwhile, this study showed that gut microbiota diversity was notably recovered, suggesting that GP may exert hepatoprotective effects mainly by changing the Firmicutes-to-Bacteroidetes ratio, while changing the amounts of several bacterial species. However, since the host genotype can also influence gut microbiota structure, it may not be appropriate to apply these results directly to humans.

\section{Conclusions}

Overall, this work suggests that structural changes in the gut microbiome after treatment with the Chinese herbal Medicine GP contribute to NAFLD amelioration. Specifically, GP administration resulted in enriched beneficial bacteria and suppressed pathogenic bacteria in the gut. Although it remains uncertain whether GP-associated gut microbiome alterations directly contribute to improving liver structure and function in NAFLD, this study provides necessary evidence demonstrating the involvement of the gut microbiota. Further studies are needed to clarify the detailed mechanisms. One of them could be faecal transplant. The effect of transfer of gut microbiota from GP-administered rats to NAFLD rats without any treatments should be analyzed to prove the significant association between the suppressive effect of GP on NAFLD and the changes in the composition of gut microbiota. Collectively, our results show GP as a potential microecological modulator for treating NAFLD.

\section{Supplementary information}

Supplementary information accompanies this paper at https://doi.org/10. 1186/s12906-020-2835-7.

Additional file 1: Table S1. GP prevents HFD-induced Non-alcoholic fatty liver disease (NAFLD) in rats Table S2. GP prevents HFD-induced 
inflammation in rats. Table S3. GP prevents HFD-induced inflammation in rats. Table S4. GP prevents HFD-induced insulin resistance in rats. Table S5. Primer sequences for DNA sequencing.

\section{Abbreviations}

ALT: Glutamic-pyruvic transaminase; AST: Glutamic oxalacetic transaminase; CVD: Cardiovascular disease; DLPC: Dilinoleoyl phosphatidylcholine; FBG: Fasting blood glucose; FINS: Fasting serum insulin; GP: Gynostemma pentaphyllum; HDL-C: High density lipoprotein cholesterol; HOMAIR: Homeostasis model assessment of insulin resistance; IL-1 B: Interleukin-1beta; IL-6: Interleukin-6; IR: Insulin resistance; LDL-C: Low density lipoprotein cholesterol; MetS: Metabolic syndrome; NAFLD: Nonalcoholic fatty liver disease; NASH: Non-alcoholic steatohepatitis; T2D: Type 2 diabetes; TC: Total cholesterol; TG: Triglycerides; TLR-4: Toll-like receptor 4; TNF-a: Tumor necrosis factor-alpha; WB: Western blotting

\section{Acknowledgements}

Thanks for Prof. Kungen Wang, Dr. Jing Guo and Dr. Xinjun Hu for helpful discussions.

\section{Authors' contributions}

SSS and BL designed the present study. TYZ, PC and JF assisted in the animal experiments and high throughput sequencing. The data analysis and original draft were conducted by SSS.All authors reviewed the results and approved the final version of the manuscript.

\section{Funding}

This study was supported by the National Natural Science Foundation of China, No.81503527; the Chinese Postdoctoral Science Foundation, No.2016 M601981; and the Item of Zhejiang Provincial TCM Sci-tech Plan, No.2017ZA045. The funders had no role in study design, data collection and analysis, decision to publish, or preparation of the manuscript.

\section{Availability of data and materials}

The datasets used and/or analysed during the current study are available from the corresponding author on reasonable request.

\section{Ethics approval and consent to participate}

The rats were bought from Zhejiang Laboratory Animal Center (Production License Number: SCXK (Zhe)2014-0001, Use License Number: SYXK (Zhe)2014-0008). Animal experiments were approved and performed in accordance with the guidelines of the Ethics Committee of the first affiliated hospital of Zhejiang Chinese medical University, Hangzhou, China (2017-k098).

\section{Consent for publication}

Not applicable.

\section{Competing interests}

The authors declare that they have no conflict of interest.

\section{Author details}

'Department of Healthcare Management, The First Affiliated Hospital of Zhejiang Chinese Medical University, Hangzhou 310006, China. ${ }^{2}$ School of Life Sciences and Biotechnology, Shanghai Jiao Tong University, Shanghai 200240, China. ${ }^{3}$ Laboratory Animal Centre, Zhejiang Academy of Medical Science, Hangzhou 310000, China. ${ }^{4}$ Department of Gastroenterology, The First Affiliated Hospital of Zhejiang Chinese Medical University, 54 Youdian Road, Zhejiang 310006, Hangzhou, China.

\section{Received: 21 March 2019 Accepted: 27 January 2020}

Published online: 05 February 2020

\section{References}

1. Lonardo A, Byrne CD, Caldwell SH, Cortez-Pinto H, Targher G. Global epidemiology of nonalcoholic fatty liver disease: meta-analytic assessment of prevalence, incidence, and outcomes. Hepatol. 2016;64:1388-9.

2. Byrne CD, Targher G. NAFLD: a multisystem disease. J Hepatol. 2015;62:547-64.

3. Than NN, Newsome PN. A concise review of non-alcoholic fatty liver disease. Atheroscler. 2015;239:192-202.
4. Rinella ME. Nonalcoholic fatty liver disease: a systematic review. JAMA. 2015; 313:2263-73.

5. Lonardo A, Ballestri S, Marchesini G, Angulo P, Loria P. Nonalcoholic fatty liver disease: a precursor of the metabolic syndrome. Dig Liver Dis. 2015;47:181-90

6. Ballestri S, Zona S, Targher G, Romagnoli D, Baldelli E, Nascimbeni F, et al. Nonalcoholic fatty liver disease is associated with an almost twofold increased risk of incident type 2 diabetes and metabolic syndrome. Evidence from a systematic review and meta-analysis. J Gastroenterol Hepatol. 2016;31:936-44.

7. Adams LA, Anstee QM, Tilg H, Targher G. Non-alcoholic fatty liver disease and its relationship with cardiovascular disease and other extrahepatic diseases. Gut. 2017;66:1138-53.

8. Satapathy SK, Sanyal AJ. Epidemiology and natural history of nonalcoholic fatty liver disease. Semin Liver Dis. 2015;35:221-35.

9. Younossi ZM, Blissett D, Blissett R, Henry L, Stepanova M, Younossi Y, et al. The economic and clinical burden of nonalcoholic fatty liver disease in the United States and Europe. Hepatology. 2016;64:1577-86.

10. Buzzetti E, Pinzani M, Tsochatzis EA. The multiple-hit pathogenesis of nonalcoholic fatty liver disease (NAFLD). Metab. 2016;65:1038-48.

11. Leung C, Rivera L, Furness JB, Angus PW. The role of the gut microbiota in NAFLD. Nat Rev Gastroenterol Hepatol. 2016;13:412-25.

12. Bashiardes S, Shapiro H, Rozin S, Shibolet O, Elinav E. Non-alcoholic fatty liver and the gut microbiota. Mol Metab. 2016;5:782-94.

13. Stone R. Biochemistry. Lifting the veil on traditional Chinese medicine. Science. 2008;319:709-10.

14. Yin J, Zhang H, Ye J. Traditional chinese medicine in treatment of metabolic syndrome. Endocr Metab Immune Disord Drug Targets. 2008;8:99-111.

15. Shi KQ, Fan YC, Liu WY, Li LF, Chen YP, Zheng MH. Traditional Chinese medicines benefit to nonalcoholic fatty liver disease: a systematic review and meta-analysis. Mol Biol Rep. 2012;39:9715-22.

16. Norberg A, Hoa NK, Liepinsh E, Van Phan D, Thuan ND, Jornvall H, et al. A novel insulin-releasing substance, phanoside, from the plant Gynostemma pentaphyllum. J Biol Chem. 2004;279:41361-7.

17. Hong M, Cai Z, Song L, Liu Y, Wang Q, Feng X. Gynostemma pentaphyllum attenuates the progression of nonalcoholic fatty liver disease in mice: a biomedical investigation integrated with in Silico assay. Evid Based Complement Alternat Med. 2018;2018:8384631.

18. Bae UJ, Park EO, Park J, Jung SJ, Ham H, Yu KW, et al. Gypenoside UL4-rich Gynostemma pentaphyllum extract exerts a Hepatoprotective effect on dietinduced nonalcoholic fatty liver disease. Am J Chin Med. 2018;46:1315-32.

19. Yang $\mathrm{YH}$, Yang J, Jiang $\mathrm{QH}$. Hypolipidemic effect of gypenosides in experimentally induced hypercholesterolemic rats. Lipids Health Dis. 2013:12:154.

20. Wang Z, Zhao X, Liu X, Lu W, Jia S, Hong T, et al. Anti-diabetic activity evaluation of a polysaccharide extracted from Gynostemma pentaphyllum. Int J Biol Macromol. 2019;126:209-14.

21. Muller C, Gardemann A, Keilhoff G, Peter D, Wiswedel I, Schild L. Prevention of free fatty acid-induced lipid accumulation, oxidative stress, and cell death in primary hepatocyte cultures by a Gynostemma pentaphyllum extract. Phytomedicine. 2012;19:395-401.

22. Qin R, Zhang J, Li C, Zhang X, Xiong A, Huang F, et al. Protective effects of gypenosides against fatty liver disease induced by high fat and cholesterol diet and alcohol in rats. Arch Pharm Res. 2012;35:1241-50.

23. Dajani Al, Abu Hammour AM, Zakaria MA, Al Jaberi MR, Nounou MA, Semrin Al. Essential phospholipids as a supportive adjunct in the management of patients with NAFLD. Arab J Gastroenterol. 2015;16:99-104.

24. Gundermann KJ, Kuenker A, Kuntz E, Drozdzik M. Activity of essential phospholipids (EPL) from soybean in liver diseases. Pharmacol Rep. 2011;63:643-59.

25. Xu ZJ, Fan JG, Ding XD, Qiao L, Wang GL. Characterization of high-fat, dietinduced, non-alcoholic steatohepatitis with fibrosis in rats. Dig Dis Sci. 2010; 55:931-40.

26. Hidalgo B, Irvin MR, Sha J, Zhi D, Aslibekyan S, Absher D, et al. Epigenomewide association study of fasting measures of glucose, insulin, and HOMA-IR in the genetics of lipid lowering drugs and diet network study. Diab. 2014; 63:801-7.

27. Nolan T, Hands RE, Bustin SA. Quantification of mRNA using real-time RTPCR. Nat Protoc. 2006;1:1559-82.

28. Ghaemmaghami S, Huh WK, Bower K, Howson RW, Belle A, Dephoure N, et al. Global analysis of protein expression in yeast. Nature. 2003;425:737-41. 
29. Caporaso JG, Kuczynski J, Stombaugh J, Bittinger K, Bushman FD, Costello EK, et al. QIIME allows analysis of high-throughput community sequencing data. Nat Methods. 2010;7:335-6.

30. Wang J, Tang H, Zhang C, Zhao Y, Derrien M, Rocher E, et al. Modulation of gut microbiota during probiotic-mediated attenuation of metabolic syndrome in high fat diet-fed mice. ISME J. 2015;9:1-15.

31. Zou Y, Li J, Lu C, Wang J, Ge J, Huang Y, et al. High-fat emulsion-induced rat model of nonalcoholic steatohepatitis. Life Sci. 2006;79:1100-7.

32. Hebbard L, George J. Animal models of nonalcoholic fatty liver disease. Nat Rev Gastroenterol Hepatol. 2011;8:35-44.

33. Jung UJ, Choi MS. Obesity and its metabolic complications: the role of adipokines and the relationship between obesity, inflammation, insulin resistance, dyslipidemia and nonalcoholic fatty liver disease. Int J Mol Sci. 2014;15:6184-223.

34. Henao-Mejia J, Elinav E, Jin C, Hao L, Mehal WZ, Strowig T, et al. Inflammasome-mediated dysbiosis regulates progression of NAFLD and obesity. Nat. 2012;482:179-85.

35. Dowman JK, Tomlinson JW, Newsome PN. Pathogenesis of non-alcoholic fatty liver disease. QJM. 2010;103:71-83.

36. Schnabl B, Brenner DA. Interactions between the intestinal microbiome and liver diseases. Gastroenterol. 2014;146:1513-24.

37. Chen D, Yang Z, Chen X, Huang Y, Yin B, Guo F, et al. The effect of lactobacillus rhamnosus hsryfm 1301 on the intestinal microbiota of a hyperlipidemic rat model. BMC Complement Altern Med. 2014;14:386.

38. Turnbaugh PJ, Ley RE, Mahowald MA, Magrini V, Mardis ER, Gordon JI. An obesity-associated gut microbiome with increased capacity for energy harvest. Nat. 2006;444:1027-31

39. Goodman AL, Kallstrom G, Faith JJ, Reyes A, Moore A, Dantas G, et al. Extensive personal human gut microbiota culture collections characterized and manipulated in gnotobiotic mice. Proc Natl Acad Sci U S A. 2011;108:6252-7.

40. Park SH, Huh TL, Kim SY, Oh MR, Tirupathi Pichiah PB, Chae SW, et al. Antiobesity effect of Gynostemma pentaphyllum extract (actiponin): a randomized, doubleblind, placebo-controlled trial. Obes (Silver Spring). 2014;22:63-71.

41. Xu J, Lian F, Zhao L, Zhao Y, Chen X, Zhang X, et al. Structural modulation of gut microbiota during alleviation of type 2 diabetes with a Chinese herbal formula. ISME J. 2015;9:552-62.

42. Wang Y, Tong Q, Shou JW, Zhao ZX, Li XY, Zhang XF, et al. Gut microbiotamediated personalized treatment of hyperlipidemia using Berberine. Theranostics. 2017:7:2443-51.

43. Wu TR, Lin CS, Chang CJ, Lin TL, Martel J, Ko YF, et al. Gut commensal Parabacteroides goldsteinii plays a predominant role in the anti-obesity effects of polysaccharides isolated from Hirsutella sinensis. Gut. 2019;68:248-62.

44. Chang CJ, Lin CS, Lu CC, Martel J, Ko YF, Ojcius DM, et al. Ganoderma lucidum reduces obesity in mice by modulating the composition of the gut microbiota. Nat Commun. 2015;6:7489.

45. Megalli S, Davies NM, Roufogalis BD. Anti-hyperlipidemic and hypoglycemic effects of Gynostemma pentaphyllum in the Zucker fatty rat. J Pharm Pharm Sci. 2006;9:281-91.

46. Vespasiani-Gentilucci U, Gallo P, Picardi A. The role of intestinal microbiota in the pathogenesis of NAFLD: starting points for intervention. Arch Med Sci. 2018;14:701-6

47. Doulberis M, Kotronis G, Gialamprinou D, Kountouras J, Katsinelos P. Nonalcoholic fatty liver disease: an update with special focus on the role of gut microbiota. Metab. 2017;71:182-97.

48. Jia L, Vianna CR, Fukuda M, Berglund ED, Liu C, Tao C, et al. Hepatocyte tolllike receptor 4 regulates obesity-induced inflammation and insulin resistance. Nat Commun. 2014;5:3878.

49. Miura $\mathrm{K}$, Ohnishi $\mathrm{H}$. Role of gut microbiota and toll-like receptors in nonalcoholic fatty liver disease. World J Gastroenterol. 2014;20:7381-91.

50. lacono A, Raso GM, Canani RB, Calignano A, Meli R. Probiotics as an emerging therapeutic strategy to treat NAFLD: focus on molecular and biochemical mechanisms. J Nutr Biochem. 2011;22:699-711.

51. Murphy EF, Cotter PD, Healy S, Marques TM, O'Sullivan O, Fouhy F, et al. Composition and energy harvesting capacity of the gut microbiota: relationship to diet, obesity and time in mouse models. Gut. 2010;59:1635-42.

52. Kim DH, Kim H, Jeong D, Kang IB, Chon JW, Kim HS, et al. Kefir alleviates obesity and hepatic steatosis in high-fat diet-fed mice by modulation of gut microbiota and mycobiota: targeted and untargeted community analysis with correlation of biomarkers. J Nutr Biochem. 2017;44:35-43.

53. Musso G, Gambino R, Cassader M. Interactions between gut microbiota and host metabolism predisposing to obesity and diabetes. Annu Rev Med. 2011;62:361-80
54. Boursier J, Mueller O, Barret M, Machado M, Fizanne L, Araujo-Perez F, et al. The severity of nonalcoholic fatty liver disease is associated with gut dysbiosis and shift in the metabolic function of the gut microbiota. Hepatol. 2016:63:764-75.

\section{Publisher's Note}

Springer Nature remains neutral with regard to jurisdictional claims in published maps and institutional affiliations.
Ready to submit your research? Choose BMC and benefit from:

- fast, convenient online submission

- thorough peer review by experienced researchers in your field

- rapid publication on acceptance

- support for research data, including large and complex data types

- gold Open Access which fosters wider collaboration and increased citations

- maximum visibility for your research: over $100 \mathrm{M}$ website views per year

At BMC, research is always in progress.

Learn more biomedcentral.com/submissions 